\title{
Voice over Wireless LAN via Wireless Distribution System*
}

\author{
Ping Chung Ng, Soung Chang Liew, and Wei Wang \\ Department of Information Engineering \\ The Chinese University of Hong Kong \\ \{pcng3, soung,wwang2\}@ie.cuhk.edu.hk
}

\begin{abstract}
Today, most Wireless LANs (WLAN) are connected to the backbone via a wired distribution system (DS). A Wireless distribution system (WDS) provides quick and easy networking in circumstances that require temporary network services or when cabling is difficult, for example, in open area conversations, disasters and military usages. WDS can be used to extend the range of wireless service coverage. Transmitting Voice-over-IP (VoIP) traffic over WDS to a WLAN in the straightforward way may fail to fulfill the tight delay requirement, and may severely limit the system capacity. A multiplex-multicast (M-M) scheme previously proposed by us [1] can improve the VoIP capacity in a WLAN operating as an infrastructure basic service set (BSS) by close to $100 \%$. This paper extends the M-M scheme for WLAN connected via a WDS. Specifically, we show that given a fixed delay requirement, the $M-M$ scheme can increase the number of supportable VoIP sessions by ten times as compared to ordinary VoIP transmission over the WDS. In addition, with 22 VoIP sessions (max limit in an 802.11b infrastructure BSS connected via wired DS), the M-M scheme can achieve acceptable packet delays, (average of $27.1 \mathrm{~ms}$ and standard deviation of $7.5 \mathrm{~ms}$ ) in a 10-hop WDS. Last but not least, we show that the use of packet scheduling in conjunction with the M-M scheme can further double the VoIP capacity while extending voice-service coverage at the same time.
\end{abstract}

\section{BACKGROUND}

Conventional VoIP scheme transmits packets to/from each client individually over the wireless channel in a WLAN. Since each VoIP packet is a very small packet (typically 10-30 Bytes), the overhead at the PHY and MAC layers becomes quite significant, substantially reducing efficiency. Further exacerbating the situation is the large number of uncoordinated VoIP packets from different sessions that may contend with each other for channel access. The net effect is that the number of VoIP sessions (VoIP capacity) that can be supported becomes severely limited and the tight delay required for good voice service may not be achievable [2]. A multiplex-multicast (M-M) scheme previously proposed by us [1] can improve the VoIP capacity in a WLAN operated as an infrastructure basic service set (BSS) by close to $100 \%$. The main idea of the M-M scheme is to combine downstream VoIP packets into a single packet for multicast over the BSS. This reduces the overhead of multiple VoIP packets, and it has been shown that the VoIP capacity can be improved by close to $100 \%$. As illustrated in Fig. 1, in the M-M scheme, a multiplexer (MUX) at the voice gateway combines multiple VoIP packets into a single multiplexed packet [3], then multicasts the multiplexed packet

* This work is sponsored by the Areas of Excellence scheme established under the University Grant Committee of the Hong Kong Special Administrative Region, China (Project Number AoE/E-01/99). to the wireless end stations through the AP using a multicast IP address. The demultiplexer in each end station extracts its respective data and forwards them to the VoIP application. Reference [1] provided an in-depth analysis of the VoIP capacity. For GSM 6.10, the M-M scheme can support 22 simultaneous voice sessions. This nearly doubles the capacity of 12 in the conventional VoIP scheme [4] for the same delay and packet loss rate requirements.

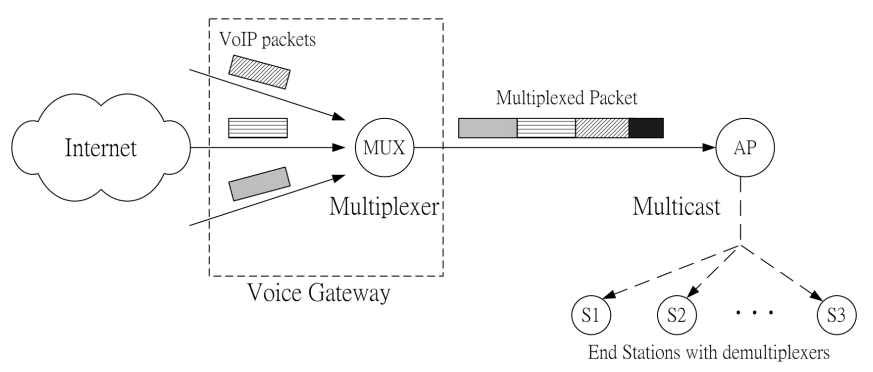

Figure 1. The M-M Scheme in Infrastructure BSS

\section{THE M-M SCHEME IN WDS}

A Wireless Distribution System (WDS) provides quick and easy network setup in areas that require temporary network services or where cabling is difficult, for example, in open areas, disaster areas, or battlefields. A WDS is basically a wireless multi-hop network, through which WLANs can be connected to the wired backbone. As shown in Fig. 2, the wireless multi-hop network comprises a number of interconnected APs, while the infrastructure BSS comprises an $\mathrm{AP}$ and client stations associated with the AP.

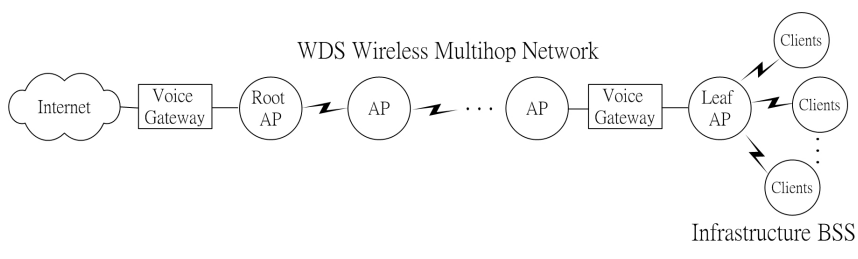

Figure 2. M-M Scheme in WDS

Reference [1] focused on the M-M scheme for VoIP over the infrastructure BSS. Here we extend the M-M scheme to provide quality of service (QoS) guarantee for VoIP over WDS. For downstream traffic, as shown in Figure $3 \mathrm{a}$, a multiplexer adjacent to the root AP multiplexes VoIP packets into a single packet and forwards it to the leaf AP through the intermediate APs. The leaf AP then multicasts the multiplexed packets to the end stations. For upstream traffic, as illustrated in Figure $3 b$, the multiplexer adjacent to the leaf AP combines 
the VoIP packets received from the end stations into a single packet and forwards it to the root AP through the WDS wireless multi-hop network. The demultiplexer adjacent to the root AP extracts the original VoIP packets and forwards them to destinations in the Internet. We separate the WDS into two parts: 1) wireless multi-hop network and 2) infrastructure BSS. We assume these two parts use different frequency channels. In other words, packet transmissions in the infrastructure BSS and wireless multi-hop network, do not interfere with each other. The reader is referred to [1] for the detailed analysis of the M$\mathrm{M}$ scheme in the infrastructure BSS. In this paper, we focus on the additional requirement of QoS guarantee in the WDS wireless multi-hop network.

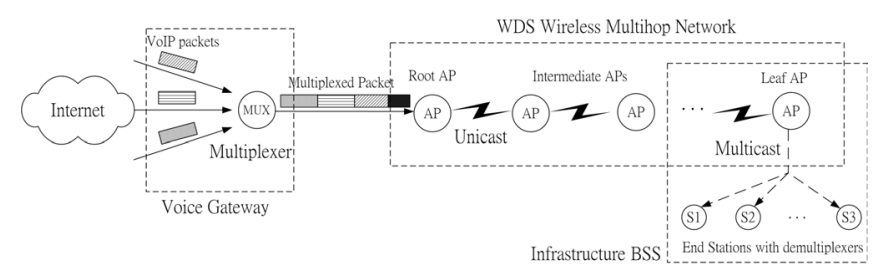

Figure 3a. M-M Scheme in WDS for Downstream VoIP Traffic

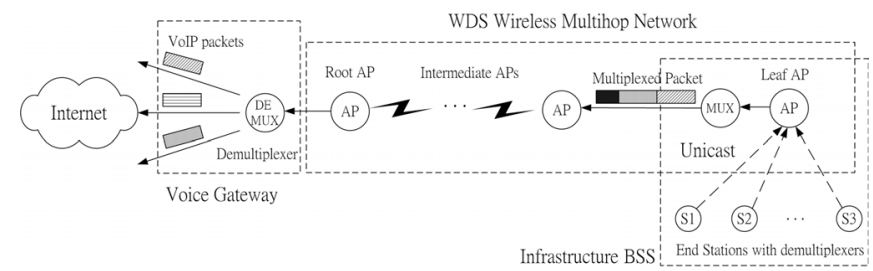

Figure 3b. M-M Scheme in WDS for Upstream VoIP Traffic

\section{QUALITY OF SERVICE (QOS)}

We quantify the QoS of VoIP with two factors: 1) packetloss rate and 2) delay performance. In this paper, we set the target packet loss rate $P_{\text {loss }}$ to be no more than $1 \%$, and the target packet delay of no more than $50 \mathrm{~ms}$. Specifically, we require the three-sigma delay (average delay $+3 *$ standard deviation of delay) to be no more than $50 \mathrm{~ms}$.

\section{A. Delay Performance}

The total delay in the WDS multi-hop network includes the transmission delay between the root $\mathrm{AP}$ and the leaf AP, $D$, as well as the multiplex delay (MUX delay) incurred at the VoIP multiplexer. MUX delays are uniformly distributed between $0 \mathrm{~ms}$ and the multiplexing interval, $M$, of the multiplexers. The average multiplexing delay, $M_{a v g}=M / 2$. Thus, the three sigma delay

$$
S=D_{a v g}+M_{a v g}+3\left(\sigma_{\text {delay }}+\sigma_{m u x}\right)
$$

where $D_{a v g}$ is the average transmission delay in the WDS multi-hop network, $\sigma_{\text {delay }}$ is the standard deviation of the transmission delay, and $\sigma_{m u x}$ is the standard deviation of the MUX delay. In this paper, we formulate our problem as maximizing the WDS voice service coverage (i.e., the number of hops in the wireless multi-hop network, $N$, which does not include the hop from the leaf AP to clients), subject to the constraints that the total delay incurred in the WDS must meet the three-sigma delay requirement, $S \leq 50 \mathrm{~ms}$, and the packet loss rate $P_{\text {loss }} \leq 1 \%$.

\section{B. Analysis of M-M Scheme}

This section provides a back-of-envelope computation for a bound on the transmission delay. Let $T$ be the average transmission time cycle of a packet in IEEE 802.11 DCF without RTS/CTS.

$$
T=\text { Packet }_{\text {multiplexed }}+S I F S+A C K+D I F S+W_{\text {avg }}
$$

where Packet multiplexed is the time to transmit the multiplexed packet, $A C K$ is the transmission time for an acknowledgement, $W_{\text {avg }}$ is the average back-off window size, SIFS is the time duration of short interframe space, and DIFS is the time duration of distributed interframe space.

Then, if there is only one packet being transmitted from the root AP (leaf AP) to the leaf AP (root AP), the journey will take $T \cdot N$ amount of time. We assume the multiplexing interval, $M$, is larger than $2 \cdot T \cdot N$. With this assumption, there will be only two packets being transmitted on the WDS multihop network at a given time in the absence of packet collisions, one in each direction. To see this, in each unit of $T$ time, one or both of the packets will make a progress of one hop: if the packets in the opposing directions are being transmitted on links that interfere with each other, then only one of them will make progress; if they are being transmitted on non-interfering links, then both of them will make progress. In any case, in $2 \cdot T \cdot N$ time, at least $2 \cdot N$ hops have been made, and this amount of "work" is that which is required to forward two packets from source to destination.

Thus, to the extend that collisions do not incur significant overhead, a rough bound for transmission delay is

$$
D_{\text {bound }}=2 \cdot T \cdot N
$$

Since the number of packets is limited to two in each MUX interval, one would expect the low-collision assumption to be valid. In addition, the above bound is obtained with the assumption that all links interfere with each other, which is quite a stringent assumption especially when $\mathrm{N}$ is large.

The above equation can be used in two ways: 1) to find a delay bound given $\mathrm{N}$; or 2 ) to find the maximum $\mathrm{N}$ given a delay requirement. For the second usage, after extensive simulations, we find that the approximation provides a good and conservative estimate for $N$ which is 1 to 2 hops less than the optimal value.

\section{Capacity Improvement}

Given a requirement that the 3-sigma delay should not exceed $50 \mathrm{~ms}$, the M-M scheme can support $N=10$ number of 
hops when there are $C=22$ number of VoIP sessions. With the same number of VoIP sessions, the conventional VoIP scheme fails to meet the delay requirement even for a one-hop WDS. In a 10-hop WDS, the conventional VoIP scheme can only support 2 VoIP sessions if the given delay requirement is to be fulfilled. This capacity is a far cry from the 22 that can be achieved with the M-M scheme. We implement the M-M scheme in ns2 [8]. Details of the simulation results are given in Table I.

TABle I. COVERAGE AND CAPACITY COMPARISON OF SimUlation Results With 50Ms 3-Sigma Delay ReQuiREMENT, $S$

\begin{tabular}{|c|c|c|c|}
\hline & M-M Scheme & \multicolumn{2}{|c|}{ Original VoIP } \\
\hline$C$ (capacity) & 22 & 2 & 22 \\
\hline$N$ (coverage) & 10 hops & 10 hops & 1 hop \\
\hline$M_{\text {avg }}$ & $10 \mathrm{~ms}$ & -- & -- \\
\hline$\sigma_{\text {mux }}$ & $5.8 \mathrm{~ms}$ & -- & -- \\
\hline$D_{\text {avg }}$ & $17.1 \mathrm{~ms}$ & $24.9 \mathrm{~ms}$ & $106 \mathrm{~ms}$ \\
\hline$\sigma_{\text {delay }}$ & $1.7 \mathrm{~ms}$ & $5.4 \mathrm{~ms}$ & $9.9 \mathrm{~ms}$ \\
\hline$S$ & $49.7 \mathrm{~ms}$ & $41.1 \mathrm{~ms}$ & $136 \mathrm{~ms}$ \\
\hline$P_{\text {loss }}$ & $<1 \%$ & $<1 \%$ & $24.4 \%$ \\
\hline
\end{tabular}

\section{Delay Improvement}

Table II lists more simulation results for a linear WDS topology with $N=4$ and $C=22$. For the M-M scheme, the three-sigma delay is $S=39.4 \mathrm{~ms}$. In contrast, for the original VoIP scheme, $S=916 \mathrm{~ms}$, which is unacceptable for real-time VoIP applications. To reduce the delay, we can decrease the number of VoIP sessions. The original VoIP can only support a maximum of 3 VoIP sessions if the 3-sigma delay requirement is to be met. An extra VoIP session (i.e. 4 sessions) increases the delay so much so that it exceeds the $50 \mathrm{~ms}$ delay requirement.

TABle II. COMPARISON OF SimUlation RESUltS FOR 4-HOP WDS

\begin{tabular}{|c|c|c|c|}
\hline & M-M Scheme & \multicolumn{2}{|c|}{ Original VoIP } \\
\hline$C$ (capacity) & 22 & 3 & 22 \\
\hline$N$ (coverage) & $4 \mathrm{hops}$ & 4 hops & 4 hops \\
\hline$M_{\text {avg }}$ & $10 \mathrm{~ms}$ & -- & -- \\
\hline$\sigma_{\text {mux }}$ & $5.8 \mathrm{~ms}$ & -- & -- \\
\hline$D_{\text {avg }}$ & $8.4 \mathrm{~ms}$ & $10.9 \mathrm{~ms}$ & $485 \mathrm{~ms}$ \\
\hline$\sigma_{\text {delay }}$ & $1.2 \mathrm{~ms}$ & $4.6 \mathrm{~ms}$ & $144 \mathrm{~ms}$ \\
\hline$S$ & $39.4 \mathrm{~ms}$ & $24.6 \mathrm{~ms}$ & $916 \mathrm{~ms}$ \\
\hline$P_{\text {loss }}$ & $<1 \%$ & $<1 \%$ & $82.8 \%$ \\
\hline
\end{tabular}

\section{SPECTRUM REUSE}

Thanks to spectrum reuse, transmitters far apart can send packets simultaneously. This means consecutive packets in the same direction separated far apart do not interfere with each other. Fig. 4 shows two possible WDS network topologies which utilize the spectrum reuse property to improve the VoIP capacity and extend coverage. In Fig. 4a, a string WDS topology can support more than one infrastructure BSS while in Fig. 4b, a lattice structured WDS can use packet scheduling to extend the service coverage. The maximum capacity of the string WDS and the extensibility of the lattice WDS are determined by the spectrum reuse factor $f$. In the string topology, $f$ represents the maximum number of infrastructure BSSs that the WDS multi-hop network can support. In lattice topology, $f$ represents the maximum number of chains within the carrier sensing range, $d$. In other words, $f$ is related to the packing density in the lattice structure. In both the string and lattice structures, $f$ relates to the number of supportable infrastructure BSSs within an area. As will be shown later, it turns out that $f$ gives an indication on how to schedule the transmission of packets to different infrastructure BSSs as to prevent collisions among them, and this in turn gives us a method to improve the total capacity of the system.

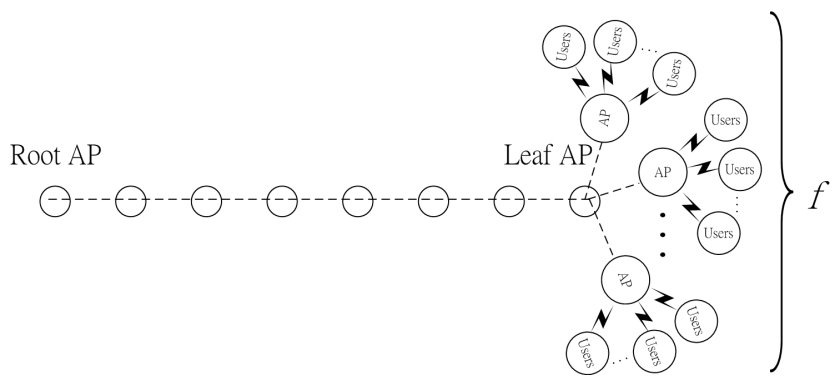

Figure 4a. String WDS with Infrastructure BSSs

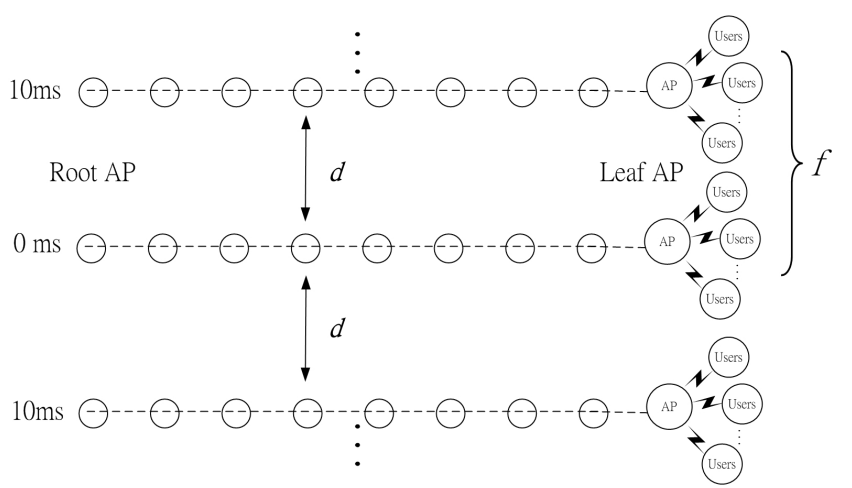

Figure 4b. Extensible Lattice WDS with Packet Scheduling

\section{A. Analysis of Spectrum Reuse Factor}

From [5], transmitters separated by more than 3 hops do not interfere with each other. When a packet meets another packet in opposite direction on the same link, they contend for channel access and must take turn to transmit. This results in extra delay. In the worst case, a packet stops and waits at the same AP for 5 consecutive transmission cycles until the packet in the opposite direction completely passes through its carrier sensing range as shown in Fig. 5. To provide sufficient delay budget to prevent subsequent packets from catching up previous packets in the same direction, we set the minimum MUX delay to be 


$$
M_{\min }=(3+5) T=8 T
$$

Consider one particular infrastructure BSS. Suppose it is the only BSS in operation. If the multiplexed packets to an infrastructure BSS use a MUX delay of $M$ rather than $M_{\text {min }}$. Then, there will be times when the root AP (leaf AP) can transmit the next packet without interfering with the earlier packets, since the earlier packets will have traveled far enough down (up) the chain. These times could be used for transmission of packets to/from other infrastructure BSSs. By scheduling the MUX intervals of different BSSs judiciously, we can increase the capacity of the system. In particular, the spectrum reuse factor is given by

$$
f=\left\lfloor\frac{M}{M_{\min }}\right\rfloor
$$

The following sections give details on how the spectrum reuse factor can be applied in string and lattice WDS.

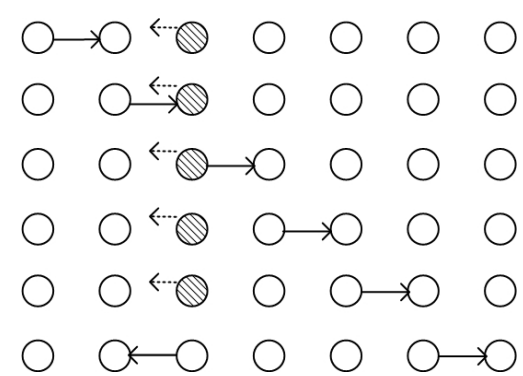

Figure 5. A packet stops and waits in the same AP for 5 consecutive transmission cycles

\section{B. Capacity Improvement in String WDS}

As shown in Table I, given a requirement that the 3-sigma delay should not exceed $50 \mathrm{~ms}$, the M-M scheme with MUX delay, $M=20 \mathrm{~ms}$ can support a 10-hop WDS. Thanks to spectrum reuse, the multiplexer delay can be reduced to $10 \mathrm{~ms}$ which in turn supports one extra infrastructure BSS, $f=2$, as shown in Fig. 4a. For example, the multiplexed packets to/from the first BSS will be transmitted from the root AP/leaf AP at time $0^{\text {th }}, 20^{\text {th }}, 40^{\text {th }} \mathrm{ms}$ and so on while those to/from the second BSS will be sent at time $10^{\text {th }}, 30^{\text {th }}, 50^{\text {th }} \mathrm{ms}$ and so on. This arrangement provides sufficient time interval to prevent subsequent packets from catching up previous ones. Simulation shows that the three-sigma delay requirement can be maintained as in Table I. In this case, the M-M scheme can achieve a further $100 \%$ improvement in VoIP capacity.

\section{Extensible Lattice Structured WDS}

In the lattice WDS, we schedule the transmission time of neighbor root APs and leaf APs to achieve the spectrum reuse property as mentioned above. The root APs and leaf APs synchronize with their neighbor APs by exchanging information. As shown in Fig. 4b, the root APs and leaf APs begin the transmissions of packets with $10 \mathrm{~ms}$ delay from its neighbors. This provides sufficient interval to ensure packets of each chain does not interfere with packets of its neighbor chains. This spectrum reuse property provides WDS with extensible voice service coverage.

\section{RELIABILITIES OF MULTICAST}

In WLAN, multicast packets are transmitted without acknowledgments. This raises a reliability concern for the M-M scheme. After extensive real-network experiments, we find that multicast packet loss rate in a 20-meter obstructed office environment is about $1-2 \%$. Translated to the WDS scenario, this means that if two nodes are separated by no more than 20meter indoor, multicast should work satisfactorily.

\section{CONCLUSION}

This paper extends our previous work on a VoIP MultiplexMulticast (M-M) scheme in infrastructure BSS to WDS. We have shown that the M-M scheme can significantly improve delay, capacity and coverage of VoIP over WDS. In addition, packet scheduling can further double the VoIP capacity and provide an extensible voice-service WDS network. We believe M-M scheme over WDS is an attractive solution for deploying voice as well as data services where quick and easy network setup is desired.

\section{REFERENCES}

[1] W. Wang, S. C. Liew, Q. Pang, V. O. K. Li "A Multiplex-Multicast Scheme that Improves System Capacity of Voice-over-IP on Wireless LAN by 100\%”, IEEE ISCC'04, Alexandria, Egypt, Jun. 2004.

[2] S. Garg and M. Kappes, "An Experimental Study of Throughput for UDP and VoIP Traffic in IEEE 802.11b Networks", IEEE WCNC'03, Vol. 3, Mar. 2003, pp.1748-1753

[3] H. P. Sze, S. C. Liew, J.Y.B. Lee, D.C.S. Yip, “A multiplexing scheme for H.323 voice-over-IP applications”, IEEE J. Select. Area Commun, Vol. 20, pp. 1360-1368, Sept. 2002.

[4] S. Garg, M. Kappes, "On the Throughput of $802.11 \mathrm{~b}$ Networks for VoIP", Technical Report ALR-2002-012, Avaya Labs, 2002. http://www.research.avayalabs.com/techreportY.html

[5] J. Li, Charles Blake et al. "Capacity of Ad Hoc Wireless Network" $A C M$ MobiCom'01, Rome, Italy, July 2001.

[6] A. Prasad, "Performance Comparison of Voice over IEEE 802.11 Scheme", IEEE VTC'99, Vol. 5, Sept. 1999, pp. 2636-2640.

[7] M. Veeraraghavan, N. Cocker, T. Moors, "Support of Voice Services in IEEE 802.11 Wireless LANs", IEEE INFOCOM'01, Vol. 1, Apr. 2001, pp.488-497.

[8] “The Network Simulator - ns2", http://www.isi.edu/nsnam/ns.

[9] G. Bianchi, "Performance Analysis of the IEEE 802.11 Distributed Coordination Function", IEEE J. Select. Area Commun, Vol. 18, No.3, pp. 535-547, Mar. 2000.

[10] F. Eshghi, A. K. Elhakeem, "Performance Analysis of Ad Hoc Wireless LANs for Real-Time Traffic", IEEE J. Select. Area Commun, Vol. 21, No. 2, Feb. 2003 